\title{
One-Directional Antenna Systems: Energy Transfer from Monomers to J-Aggregates within 1D Nanoporous Aluminophosphates
}

Rebeca Sola-Llano, ${ }^{\dagger}$ Yasuhiko Fujita, ${ }^{\ddagger, \S \odot ~ L u i s ~ G o ́ m e z-H o r t i g u ̈ e l a, ~}{ }^{\oplus \odot}$ Almudena Alfayate, ${ }^{\|}$Hiroshi Uji-i, ${ }^{\ddagger} \perp$ Eduard Fron, ${ }^{\ddagger}$ Shuichi Toyouchi, ${ }^{\ddagger}$ Joaquín Pérez-Pariente, ${ }^{||}$Iñigo López-Arbeloa, and Virginia Martínez-Martínez ${ }^{*}+$ Ð0

${ }^{\dagger}$ Departamento de Química Física, Universidad del País Vasco, UPV/EHU, Apartado 644, 48080 Bilbao, Spain

${ }^{\ddagger}$ Department of Chemistry, Katholieke Universiteit Leuven, Celestijnenlaan 200F, B-3001Heverlee, Belgium

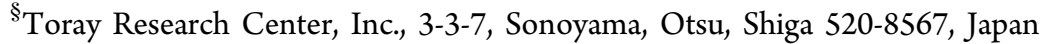

"Instituto de Catálisis y Petroleoquímica-CSIC, C/Marie Curie 2, 28049, Cantoblanco, Madrid, Spain

${ }^{\perp}$ RIES, Hokkaido University, N20W10, Kita-Ward Sapporo 001-0020, Japan

\section{Supporting Information}

ABSTRACT: A cyanine dye (PIC) was occluded into two 1D-nanopoporus $\mathrm{Mg}$ containing aluminophosphates with different pore size (MgAPO-5 and MgAPO-36 with AFI and ATS zeolitic structure types, with cylindrical channels of $7.3 \AA$ diameter and elliptical channels of $6.7 \AA \times 7.5 \AA$, respectively) by crystallization inclusion method. Different J-aggregates are photophysically characterized as a consequence of the different pore size of the MgAPO frameworks, with emission bands at $565 \mathrm{~nm}$ and at $610 \mathrm{~nm}$ in MgAPO-5 and MgAPO-36, respectively. Computational results indicate a more linear geometry of the J-aggregates inside the nanochannels of the MgAPO-36 sample than those in MgAPO-5, which is as a consequence of the more constrained environment in the former. For the same reason, the fluorescence of the PIC monomers at $550 \mathrm{~nm}$ is also activated within the MgAPO-36 channels. Owing to the strategic distribution of the fluorescent PIC species in MgAPO-36 crystals (monomers at one edge and J-aggregates with intriguing emission properties at the other edge) an efficient and one-directional

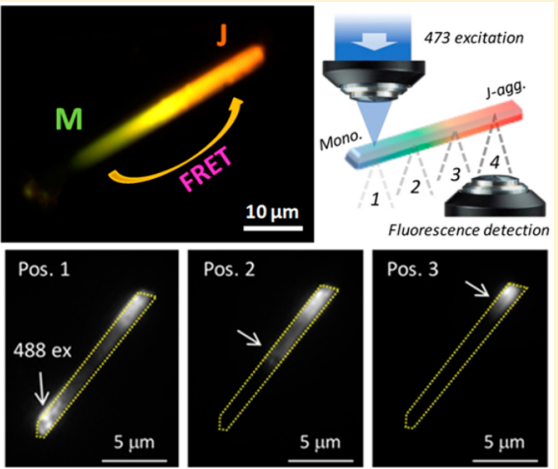
antenna system is obtained. The unidirectional energy transfer process from monomers to J-aggregates is demonstrated by remote excitation experiments along tens of microns of distance.

KEYWORDS: PIC dye, MgAPO, crystallization inclusion method, strategic distribution within nanochannels, energy transfer, unidirectional antenna effect

$\mathrm{T}$ he study and simulation of different natural systems and processes has always attracted a great interest to scientists. In this regard, an interesting challenge is the design of artificial antenna systems by mimicking the photosynthesis process, in which the sunlight is harvested and its energy is efficiently transferred to a specific reaction center where the energy conversion takes place. ${ }^{1,2}$ One of the most common mechanisms in which the energy is transferred within artificial antenna systems is the Excitation Energy Transfer process (EET), known as FRET (Fösters Resonance Energy Transfer), which consists on a nonradiative energy transfer from a donor molecule in the excited state to an acceptor molecule in the ground state that can take place over relatively long distances (Förster distances typically range from 2 to $9 \mathrm{~nm}$ ).

In this context, host-guest hybrid materials have shown appealing properties to promote FRET processes when different organic chromophores are combined within zeolitic matrices, as has been widely reported. ${ }^{2,4-8}$ Zeolitic materials based on onedimensional nanopores are indeed the best choice as host materials to obtain artificial antenna systems. In these systems, dyes absorbing and emitting in different ranges of the electromagnetic spectrum are inserted sequentially into the channels of the zeolite so that the energy can be funneled along the nanopore. In this sense, among the different compositions available for zeolitic materials, we have selected to work with aluminophosphates (AlPO) because of their ease for being synthesized as very large single crystals. However, loading of such long host systems with the photoactive species by postsynthesis (adsorption) procedures is strongly hindered by diffusion. Moreover, by this method normally the energy is transferred from the center to the edges of the channels or conversely from both edges to the center.

The crystallization occlusion method where the dyes are in situ occluded during the crystallization process represents the most convenient preparation method for a more homogeneous filling

Special Issue: Strong Coupling of Molecules to Cavities

Received: May 31, 2017

Published: October 19, 2017 

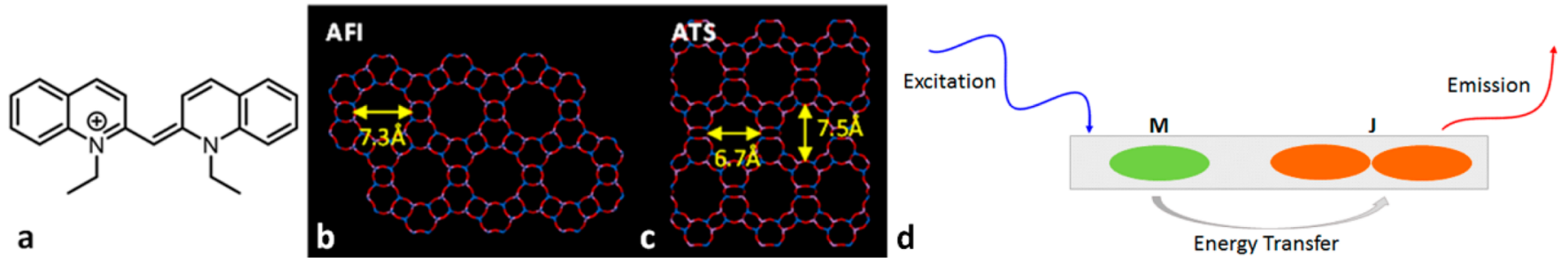

Figure 1. (a) Molecular structure of the cyanine dye 1,1'-diethyl-2,2'-cyanine, or PIC $(14.1 \AA \times 7.3 \AA \times 4.0 \AA)$. Structures and pore dimensions of the (b) AFI (MgAPO-5) and (c) ATS (MgAPO-36) frameworks. (d) Schematic representation of the dye-species distribution within MgAPO channels and the unidirectional antenna effect mediated by energy transfer process from the dye monomers to its J-aggregates.

of large crystals since traffic jam obstacles are avoided. It allows also a notable reduction of the sample preparation time since the synthesis takes place in a unique step, and a very tight fit between the molecular size of the dyes and the dimensions of the cavities of the host material that could not have been achieved otherwise. Such a tight fit prevents a posterior leakage of the dye molecules, avoiding the need for stopcock molecules. ${ }^{8-10}$ More importantly, this encapsulation approach can offer a strategic distribution of the dye-aggregates and monomers within the channels of the Mg-containing aluminophosphate MgAPO-36 (with ATS structure-type), as has been demonstrated in previous works for Pyronine Y and Acridine. ${ }^{11,12}$ In this type of framework, the crystals grow in a bouquet arrangement from the joined center of the bouquet to the ends, where apparently the dye is incorporated in a higher amount at the initial stage of crystallization. As a result, the dye is incorporated mainly in a J-type association at the initial stages of crystallization and in monomeric units at the last stages of the crystal growth; hence crystallization proceeds showing a multicolour emission characteristic of the monomers and J-aggregates. Thus, the energy transfer could occur only in one-direction, i.e. from monomers to J-aggregates, from one end to the other end of the particles. However, the FRET process between the different species occluded into the hybrid material that would take place along several microns of distance has not been experimentally evidenced yet.

In this work, new hybrid systems containing a cyanine dye, in particular 1,1'-diethyl-2,2'-cyanine, also known as pseudocyanine or PIC (Figure la) is described. The choice of this particular dye is based on: a) the perfect fit between the molecular dimensions of PIC dye and the pore size of MgAPOs structures (Figure 1) and b) the intriguing properties of J-aggregates of cyanine-like dyes, that is, high fluorescence efficiency and high exciton mobility, ${ }^{13-16}$ properties of crucial importance for an efficient light harvesting, making them very promising for artificial antenna systems. As previously studied in solution and in other media, such as solid interface, the final spectroscopic properties of these cyanine-like J-aggregates depend on the aggregation number, the degree of packing arrangement, and the dye molecular orientation. ${ }^{18-19}$ Thus, a head-to-tail arrangement is expected to be induced through the encapsulation of cyanine dyes into one-dimensional Mg-containing aluminophosphate hosts by the crystallization inclusion method, promoting the formation of nearly linear J-aggregates. In this line, two frameworks with different channel dimensions and shape were chosen, MgAPO-5 (AFI type structure with cylindrical channels of $7.3 \AA$ diameter, Figure $1 \mathrm{~b}$ ) and MgAPO-36 (ATS type structure, with elliptical channels of $6.7 \AA \times 7.5 \AA$, Figure 1c), to study the effect of the matrix on the arrangement adopted by the occluded PIC molecules and to evaluate the final photophysical properties of the different J-aggregates formed. Finally, in an attempt to experimentally demonstrate that the energy transfer takes place unidirectionally, that is, one-directionally from cyanine monomers in one end of the particles to its J-aggregates in the opposite end (Figure 1d), remote excitation experiments are performed. The one directional antenna effect along the crystals of the new PIC/MgAPO systems is also compared with previously described hybrid materials consisting of other commercial dyes (Acridine, AC, and Pyronin Y, PY) occluded within MgAPO-36. ${ }^{11,12}$

\section{EXPERIMENTAL SECTION}

Synthesis of Dye-Loaded MgAPO Materials. The Mgcontaining nanoporous aluminophosphates (MgAPO-5 and MgAPO-36) were prepared by hydrothermal synthesis using phosphoric acid (Aldrich, 85 wt \%), magnesium acetate tetrahydrate (Aldrich, $99 \mathrm{wt} \%$ ), aluminum hydroxide (Aldrich), triethylamine (TEA, Aldrich) in the case of MgAPO-5 or tripropylamine (TPA, Aldrich) in the case of MgAPO-36 as structure-directing agents, and the dye (1,1'-diethyl-2,2' -cyanine, PIC, Sigma-Aldrich). The gels have the following molar composition: (i) $0.2 \mathrm{MgO} / 1 \mathrm{P}_{2} \mathrm{O}_{5} / 0.9 \mathrm{Al}_{2} \mathrm{O}_{3} / 0.75 \mathrm{TEA} / 0.024$ $\mathrm{PIC} / 300 \mathrm{H}_{2} \mathrm{O} / 50 \mathrm{EtOH}$ for PIC/MgAPO-5 sample; (ii) 0.2 $\mathrm{MgO} / 1 \quad \mathrm{P}_{2} \mathrm{O}_{5} / 0.9 \quad \mathrm{Al}_{2} \mathrm{O}_{3} / 0.75 \mathrm{TPA} / 0.024 \mathrm{PIC} / 300 \quad \mathrm{H}_{2} \mathrm{O} / 50$ $\mathrm{EtOH}$ for PIC/MgAPO-36 sample. Note that 0.024 was set as the maximum dye amount added to the gel not to disrupt the required final phase of the crystal. Moreover, ethanol was added in order to favor the solubility of PIC in the aqueous synthesis gel. The $\mathrm{pH}$ of the synthesis gels was between 4 and 5 . The gel was heated at $180{ }^{\circ} \mathrm{C}$ under autogenous pressure and in continuous rotation for $24 \mathrm{~h}$ for MgAPO-5 sample, and statically at $180{ }^{\circ} \mathrm{C}$ under autogenous pressure for $12 \mathrm{~h}$ for MgAPO-36 sample. The solid products were recovered by filtration, exhaustively washed alternating water (at least $2 \mathrm{~L}$ ) and ethanol (at least $1 \mathrm{~L}$ ), until a colorless supernatant is recovered and dried at room temperature overnight.

Characterization. X-ray powder diffraction (XRD) was used to determine the crystalline phase obtained; XRD patterns were collected with a Panalytical X'Pro diffractometer using $\mathrm{Cu} \mathrm{K} \alpha$ radiation (Figure S1). The dye content within the solid products was determined photometrically using a double beam Varian spectrophotometer (Cary 7000), after dissolving the solid material in $5 \mathrm{M}$ hydrochloric acid and comparing the resulting solutions with standard solutions prepared from known concentrations of the dyes at the same $\mathrm{pH}$ value of the sample solutions (Figure S2).

Fluorescence images were recorded with an optical upright microscope with epi configuration (Olympus BX51) equipped with a color CCD camera (DP72, Olympus). Crystals were excited under blue light $(\lambda=470 \mathrm{~nm})$ by D470/40 Chroma a band-pass filter and the emission was collected with a E515LPv2 

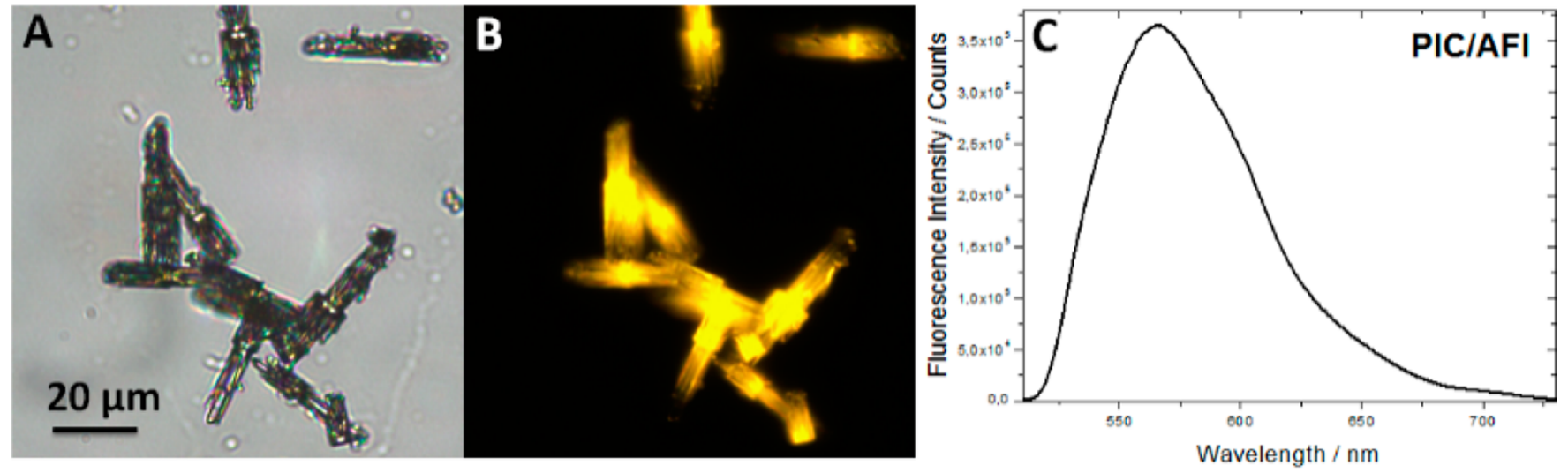

Figure 2. (A) Transmission and (B) fluorescence under blue excitation light (450-490 nm) images of the PIC/AFI particles. (C) Fluorescence emission spectrum recorded from single particles.

Chroma cutoff filter. Fluorescence spectra of single particles were recorded by a fiber coupling from Olympus microscope to Edinburgh Instruments spectrofluorimeter (model FLSP920).

Single particle fluorescence study was conducted on a homebuilt fluorescence microscope equipped with two objective lenses: a sample was placed between top and bottom objectives, respectively, for excitation and detection of fluorescence. CW laser light $(375 / 473 / 488 \mathrm{~nm})$ was introduced to the top objective (Nikon, PlanFluor, x20, NA 0.85) and was focused onto the sample. Fluorescence from the sample was collected by the bottom objective (Nikon, PlanApo, x60, NA 0.95) and was guided to a spectrograph (Andor, SR303i) equipped with EMCCD detector (Andor, Newton 970) after passing through a pinhole $(100 \mu \mathrm{m})$, or to EM-CCD detector (Andor iXon 897) for fluorescence imaging. A suitable filter (375 excitation: Thorlabs, FEL0400, 473 excitation: Chroma, HQ485LP, 488 excitation: Chroma, HQ500LP) was used in front of the spectrograph/imaging CCD to further suppress the excitation light.

Computational Details. The computational methodology employed to study the occlusion of the cyanine dye within AFI and ATS frameworks was based on DFT $+\mathrm{D}$ plane-wave simulations, as implemented in CASTEP in Materials Studio software. $^{20}$ The geometry of the zeolite structures were taken from the Material Studio database. Molecular structures and the energies of the dye molecules with the framework were obtained using the PBE exchange functional, and the Grimme dispersion correction term, and an energy cutoff of $489.8 \mathrm{eV}$. Periodic boundary conditions (PBC) were applied in all the calculations. Supercells with 3 (for AFI) and 5 (for ATS) unit cells along the channel direction were used as framework models for loadings of 1 molecule per supercell, while 6 and 10 primary unit cells were used for the study of loadings of 2 molecules per supercell. The cyanine dye molecules were manually loaded in the required configuration and geometry-optimized. Relative energies (per dye molecule) were obtained with respect to the most stable case. Interaction energies were calculated by subtracting the energy of the molecules in vacuo to the total energy of the system; all the energy values are given in $\mathrm{kcal} / \mathrm{mol}$ per dye molecule.

\section{RESULTS AND DISCUSSION}

As stated before, the PIC dye (Figure 1a) was occluded into two inorganic 1D-frameworks with different pore size and shape: MgAPO-5 (AFI structure-type), formed by 12-ring cylindrical channels of $7.3 \AA$ diameter; ${ }^{21-23}$ and MgAPO-36 (ATS structure-type), also formed by 12-ring channels but with an elliptical shape and slightly smaller pore dimensions of $6.7 \AA ̊ x 7.5$ $\AA$ (Figure $1 \mathrm{~b}, \mathrm{c}){ }^{11,12,21}$

In the first attempt, PIC was occluded into the larger pores of the AFI structure. Pure AFI phase with a dye loading of 2.94 mmol PIC per $100 \mathrm{~g}$ sample powder (Figure S2) was achieved from the hydrothermal synthesis. The hybrid material is composed of rod-shaped particles of tens of microns, which show yellow fluorescence only in the central part because of the apparent growth of AFI crystals from the center to both edges. This fluorescence emission band with maximum at $565 \mathrm{~nm}$ (Figure 2) was ascribed to PIC J-aggregates, which appears slightly blue-shifted with respect to the J-band previously detected in aqueous solution (centered at $572 \mathrm{~nm}$, see Figure S3 in Supporting Information). ${ }^{16}$ Note that the displacement of the bands is a typical feature for encapsulated dyes into highly constricted environments, ${ }^{12}$ which in this case will affect also the stacking. The result is in agreement with previous results of similar hybrid systems prepared also through the crystallization inclusion method, with J-aggregates located at the crystal growth center. $^{11,12}$ Similarly to former dye/MgAPO systems already described with AC and PY dyes, ${ }^{11,12}$ the respective monomers would be likely located at the other end of the rods, but unfortunately, they are not fluorescent in this sample. ${ }^{16}$ This fact is a typical characteristic of dyes as PIC with flexible molecular structures, since the molecular motions induce important nonradiative deactivation pathways. Thus, this result indicates that the rigidity imposed by the AFI matrix to the PIC monomers is not strong enough to switch on its emission.

Therefore, we next considered the ATS framework to occlude the PIC cyanine in a more constrained environment (because of the smaller channels, Figure 1). The synthesis experiments rendered in this case a material composed mainly of the ATS phase but with minor impurities of the AFI phase (Figure S1 in the Supporting Information) and with a dye-loading of 1.52 mmol PIC per $100 \mathrm{~g}$ of sample. In this case, the dye uptake represents half of the loading (Figure S2) obtained previously for $\mathrm{PIC} / \mathrm{AFI}$ sample $(2.94 \mathrm{mmol} / 100 \mathrm{~g}$ of sample), which is likely due to the smaller pores with elliptical shape of this host.

Indeed, computational results suggest a slightly better fitting of the dye molecules within the AFI channels with respect to ATS due to the larger pores of cylindrical shape of the former host (Figure S4). This better fitting in AFI provides an explanation for the higher loading of PIC found experimentally in this sample (PIC/AFI). 

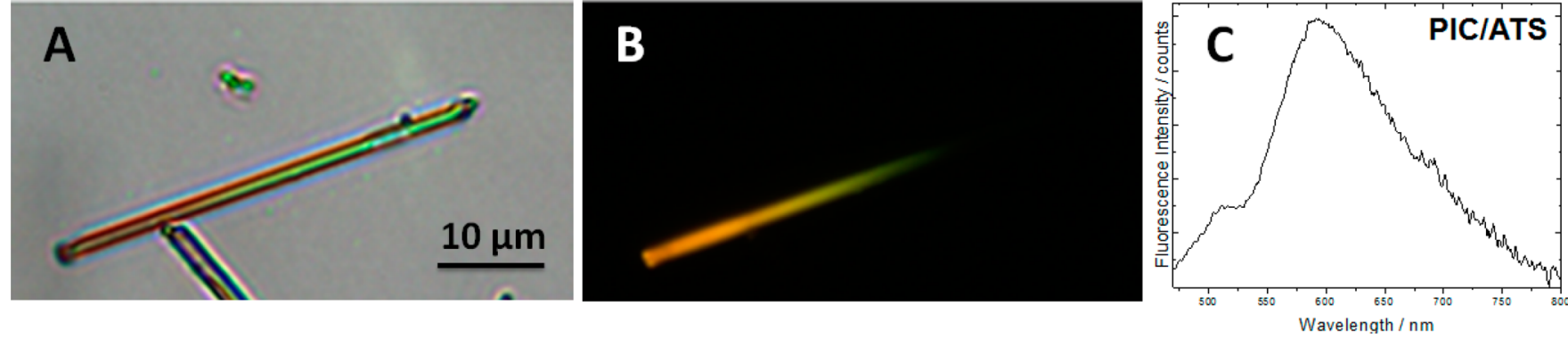

Figure 3. Transmission (A) and fluorescence under blue excitation light (450-490 nm) (B) images of a PIC/ATS needle-shaped particle (polarization direction of emission light was set along to the channels of the longer needle, that is, parallel to its long axis). (C) Fluorescence spectrum of the PIC/ATS sample recorded from bulk powder $\left(\lambda_{\text {exc }}: 450 \mathrm{~nm}\right)$.
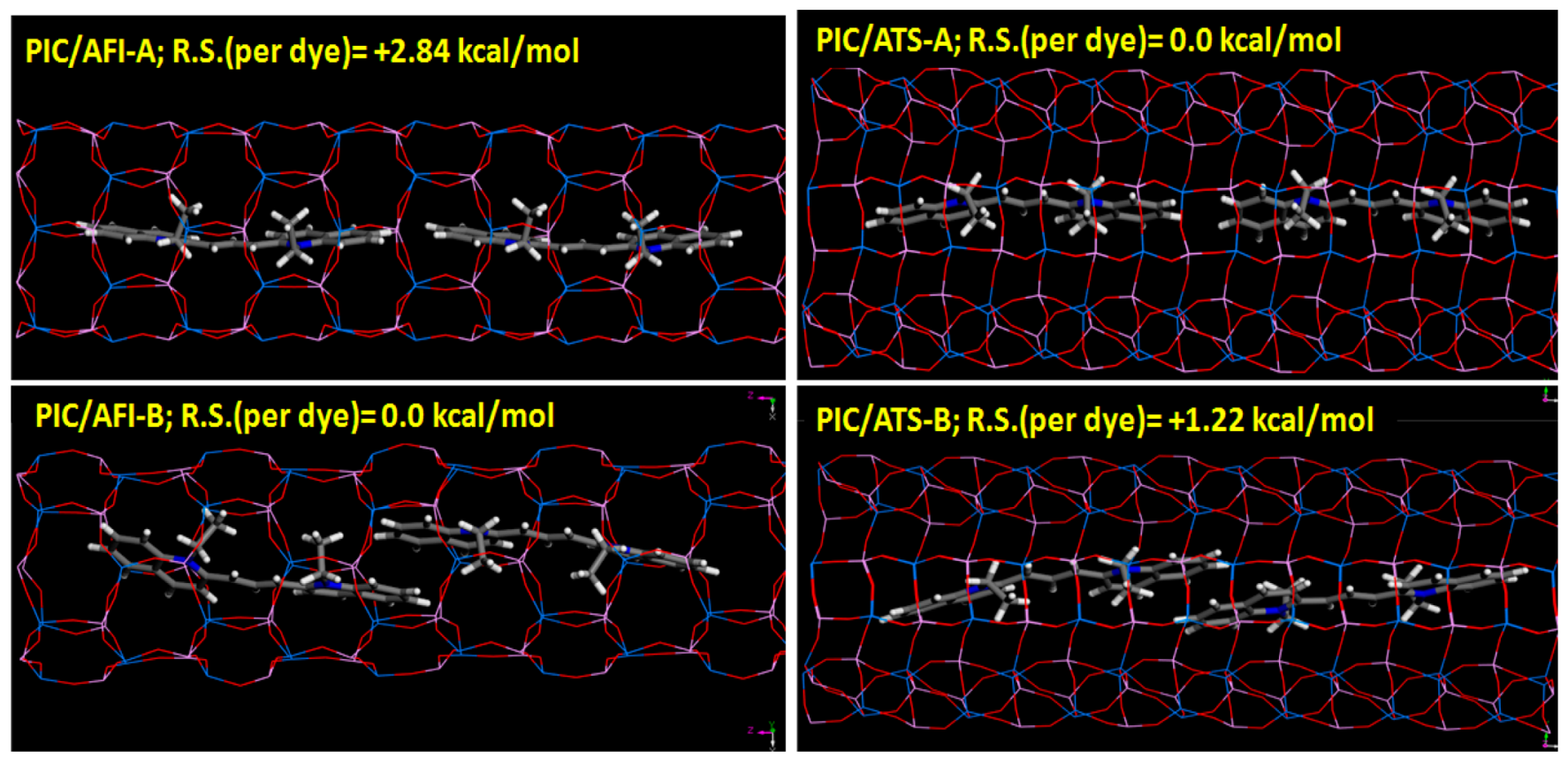

Figure 4. Different J-aggregate geometries (top: in line; bottom: displaced sandwich-like) in AFI (left) and ATS (right) frameworks and the corresponding calculated relative stability (R.S.) energies per dye.

Note here that, according to the microscopy images (Figure 3), PIC/ATS sample shows needle-shaped PIC/ATS crystals with relatively high probability (55\% of the total ATS crystals) of finding a dye-species distribution similar to that found in previous works, ${ }^{11,12}$ that is, with dye J-aggregates placed at one edge of the particles and monomers in the opposite end, likely as a consequence of ATS crystal growth from end to end of the particles. Consequently, a darker reddish color is noticed in one edge of the needle in the transmission image (Figure 3A), indicative of a higher amount of PIC occluded, which corresponds to the orange area in the fluorescence image (Figure 3B), giving an emission band centered at $610 \mathrm{~nm}$ (Figure 3C). At the opposite end of the particles, where a less amount of dye is occluded, a reminiscent green emission is apparent in the respective fluorescence image (Figure 3B). Thus, a gradual emission color change from orange to green is detected in single particles from edge to edge of the crystals, which is attributed to a strategic distribution of J-aggregates at one end and monomers at the other end of the needles, respectively. The presence of such gradient in the PIC loading is probably due to the variation of the concentration of the dye in the solution as the crystallization proceeds. At the beginning of crystallization, the large concentration of the dye produces its aggregation, but as it is depleted in the solution due to its incorporation into the growing crystals, it is occluded as monomeric form in later stages of the crystallization.

In general terms, the emission intensity in PIC/ATS particles is relatively lower than in PIC/AFI sample. However, it is important to note that PIC monomers turn out to be fluorescent into the ATS framework, giving green fluorescence (Figure 4B), that can be also seen as a shoulder at around $520 \mathrm{~nm}$ in the emission spectrum of the bulk powder (Figure 4C). This result is attributed to the tight confinement into the ATS matrix that reduces the probability of monomers deactivation through nonradiative pathways, ${ }^{24}$ which represents a novel finding for PIC dye.

Interestingly, PIC J-aggregates show different emission color in each framework: a yellow emission characterized by a fluorescence band at $565 \mathrm{~nm}$ in the AFI host, and a less intense and red-shifted emission, characterized by an orange fluorescence centered at $610 \mathrm{~nm}$ in the ATS structure. These differences are ascribed to J-aggregates adopting a different geometry in each host, and it can be explained by means of the Exciton Theory (Figure S5). ${ }^{25,26}$ According to this theory, the angles between the molecules forming the aggregates determine the spectral changes. In this context, the more in-line the J-aggregate, the 
higher energy splitting of the J-band with respect to the monomer band is expected, and therefore a higher bathochromic shift will be observed in the emission band. Consequently, we can assume that J-aggregates formed in the PIC/ATS sample are more linear than those in the PIC/AFI case, and thus, the spectral shift of the emission band to the red edge is larger.

Molecular simulations were performed in order to confirm the above statements about the geometry of the J-aggregates inside the nanochannels of the two host structures. To simulate the more stable geometry, two PIC molecules were loaded into the frameworks forming J-aggregates in two possible geometries (Figure 4), that is, as perfect in-line head-to-tail J-dimers (top: A) or as displaced J-dimers in a sandwich-like disposition (bottom: B). The calculated relative stabilities and interaction energies clearly show that cyanine dimers are more stable in a displaced Jtype configuration in the AFI channels, while the in-line Jgeometry is the most stable configuration for PIC J-aggregates confined within ATS. Again, this is due to the larger cross-section of the AFI channels, whose dimensions are suitable to host the cyanine dyes enabling $\pi-\pi$ interactions between the aromatic rings that stabilizes the displaced J-type configuration. Note here that obviously pure $\mathrm{H}$-aggregates (nonemissive and efficient fluorescence quenchers) cannot be formed even in the larger AFI channels, because the presence of the ethyl substituents in the dye (Figure 1a) prevents this configuration. The smaller crosssection in one dimension of the ATS channels causes a steric hindrance between the aromatic rings of the dye molecules, inducing its disposition as pure in-line head-to-tail J-aggregates.

Regarding the strategic distribution of the fluorescent species into MgAPO-36 observed in Figure 3B, and in order to obtain experimental evidence of the FRET process from monomers to aggregates in this PIC/ATS system, further single particle microscopy experiments were performed. By exciting at the monomer region (position 1 in Figure 5a), a fluorescence band centered at $550 \mathrm{~nm}$ was observed, while exciting at the Jaggregate region (position 4 in Figure 5a), the fluorescence peak
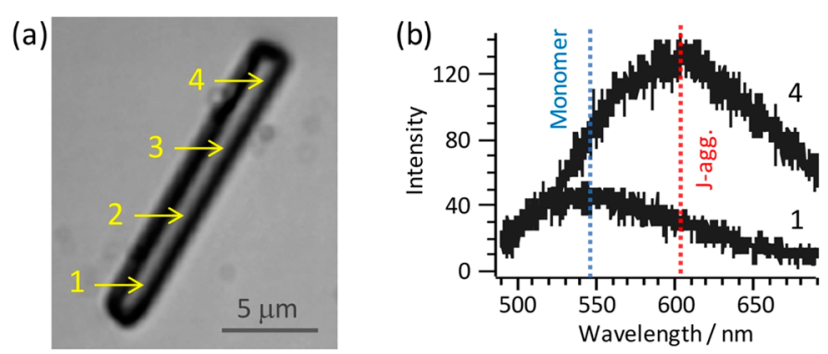

(c)
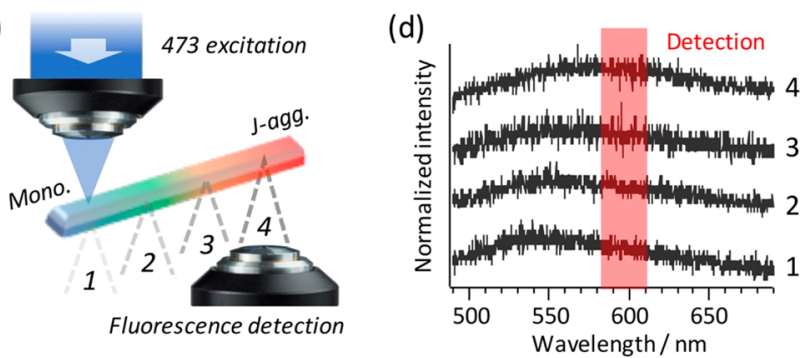

Figure 5. (a) Transmission image of a PIC/ATS crystal. (b) Fluorescence spectra obtained at positions 1 and 4. (c) Schematic illustration of remote excitation spectroscopy. (d) Normalized fluorescence spectra at positions 1-4 obtained with the remote excitation spectroscopy by focusing continuous laser light $(473 \mathrm{~nm})$ with $120 \mathrm{~kW} / \mathrm{cm}^{2}$ power density at position 1 . was shifted to $610 \mathrm{~nm}$ with an intensity about $3 \times$ higher than that of the monomeric band (Figure $5 b$ ). As a consequence of the enhanced fluorescence properties of the J-aggregates of the cyanine dyes with respect to their monomers in terms of fluorescent emission intensity, their emission band appears nearly isolated from that ascribed to monomeric species (normalized fluorescence spectra measured at positions 1-4 can be seen in Figure S6). This is not the case for the previously characterized samples ${ }^{11,12}$ where the emission of the monomers is observed even at much lower concentration in comparison to the J-aggregates (Figure S7 in Supporting Information). More importantly, this gradual red-shift in the fluorescence peak from 550 to $610 \mathrm{~nm}$ measured at positions $1-4$ is also visualized in the remote excitation experiments (Figure $5 \mathrm{~d}$ ), where the excitation was fixed at the monomer region (position 1 in Figure 5a) while the detection point is displaced toward the other end of the particle, as illustrated in Figure 5c. This result indicates that the energy transfer from monomers to J-aggregates is actually taking place along the crystal.

Indeed, the energy transfer process in the PIC/ATS system, i.e. the antenna effect, is further experimentally demonstrated by measuring fluorescence images (Figure 6b); these are compared with our previously reported crystals (Figure $6 \mathrm{~d}, \mathrm{f}, \mathrm{h}, \mathrm{j}$ ). Figure $6 \mathrm{~b}$ shows fluorescence images under excitation with continuous wave $488 \mathrm{~nm}$ laser light at different positions from monomers to $\mathrm{J}$-aggregates (indicated by an arrow in each image) from the bottom to the top of another PIC/ATS crystal, as shown in Figure 6a. At excitation position 1, the laser was focused on the region where monomeric species are found and, as can be seen, fluorescence emission is detected in the whole particle. On the other hand, by moving the laser focus toward the J-aggregate region (top region), the intense fluorescence in the monomer region is no longer seen, while the J-aggregate region always shows bright fluorescence (Figure $6 \mathrm{~b}$ position 2 ). These results indicate that energy migration is in fact one-directional in the PIC/ATS system, taking place from monomers at one end of the particles to J-aggregates at the opposite end, along tens of microns. Moreover, when the laser is focused at the top end (Figure $6 \mathrm{~b}$ position 3 ), there is no fluorescence detected at the bottom end, indicating that a dielectric wave-guiding effect of the crystal itself does not play a dominant role.

Besides, by comparing the fluorescence images with our previously reported crystals (crystals containing pyronin Y (PY) and acridine (AC) into the ATS framework), ${ }^{11,12}$ the newly synthesized PIC/ATS system gives much more efficient antenna effect (Figures 6 and S8). Indeed, in the previous AC and PY containing ATS samples, the energy transfer does not reach the opposite end in any of the samples. In the best case, for AC-PY/ ATS samples, the antenna effect reaches just over $5 \mu \mathrm{m}$ (i.e., Figure $6 \mathrm{f}$ position 1 and Figure $6 \mathrm{~h}$ position 2). Considering these results, we conclude that our newly synthesized PIC/ATS hostguest system yields a very efficient and long-range onedirectional artificial antenna system through successive FRET processes taking place over tens of microns, mainly as a consequence of the interesting properties of the J-aggregates of PIC dye.

\section{CONCLUSIONS}

MgAPO-5 (AFI) and MgAPO-36 (ATS) host structures have demonstrated to induce two geometrically different J-aggregate species of the $1,1^{\prime}$-diethyl-2,2'-cyanine (PIC) dye, occluded within the frameworks through the crystallization inclusion method. In the ATS structure-type, the narrower size and 
(a)

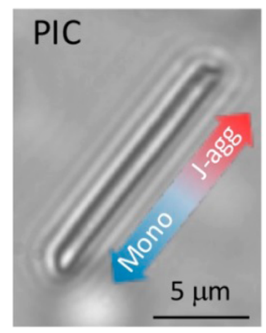

(c)

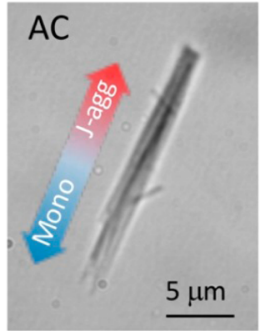

(e)

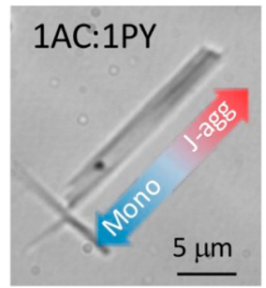

(g)

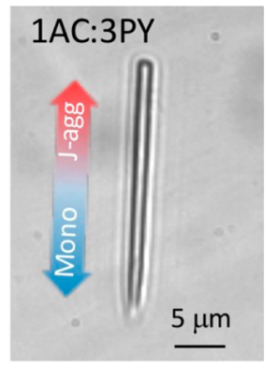

(i)

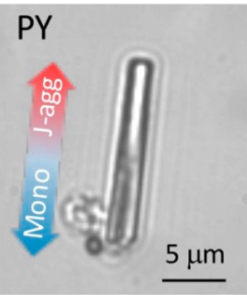

(b)
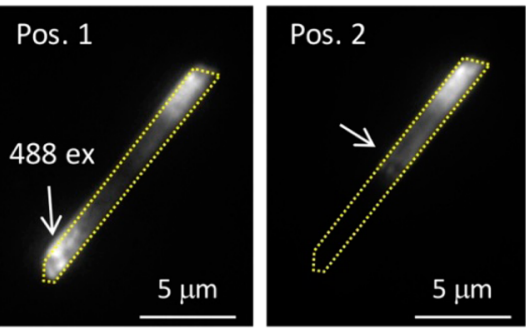

(d)

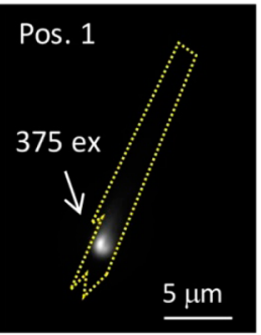

(f)

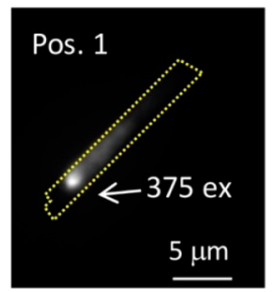

(h)

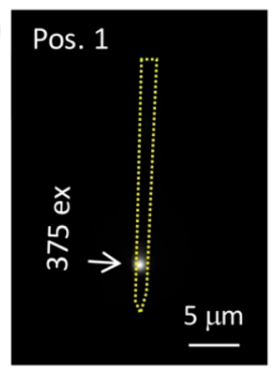

(j)

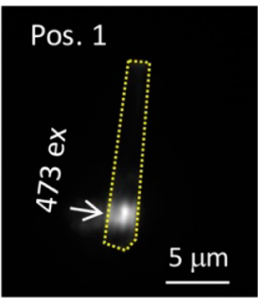

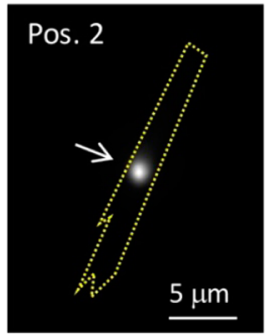
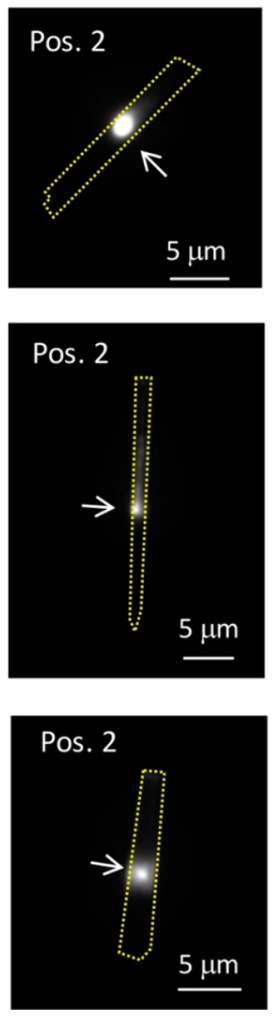
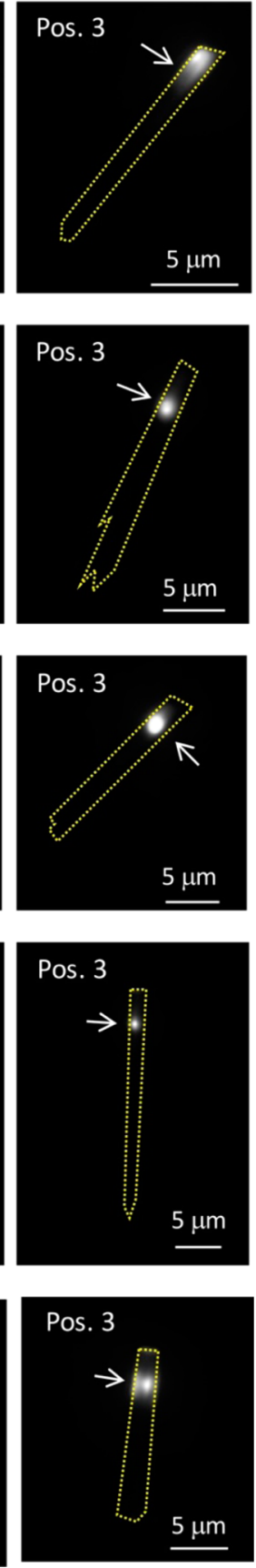

Figure 6. Transmission and fluorescence images collected from 3 different excitation positions on PIC/ATS (a, b), AC/ATS (c, d), 1AC:1PY/ATS (e, f), 1AC:3PY/ATS (g, h), and PY/ATS (i, j) crystals. Excitation laser wavelength/positions are indicated in each image.

elliptical shape of the nanopores promotes the formation of more linear J-aggregates with a band centered at $610 \mathrm{~nm}$. Moreover, the fluorescence from PIC monomers at around $520 \mathrm{~nm}$ is also observed in the ATS framework (but not in AFI), as a consequence of the more constrained environment of the ATS nanochannels, which limits the flexibility of the molecules, and consequently reduces the probability of nonradiative deactivation. Interestingly, owing to the strategic distribution of the different species (monomers at one edge and J-aggregates at the other end with crucial photophysical properties) achieved with this synthetic procedure, an efficient antenna system has been obtained were the energy transfer takes place exclusively along one direction, that is, from end to end of the micrometer-sized crystals, a fact that is experimentally evidenced through remote excitation experiments.

\section{ASSOCIATED CONTENT}

\section{S Supporting Information}

The Supporting Information is available free of charge on the ACS Publications website at DOI: 10.1021/acsphotonics.7b00553.

XRD patterns of the PIC/ATS and PIC/AFI powder, absorption spectra of dissolved crystals, computational results of PIC/AFI and PIC/ATS, spectra of PIC species in solution, schematic representation of the exciton splitting of the electronic states of the different geometries of PIC J-aggregates, Transmission images, fluorescence 
spectra measured at different positions and wide field images of PIC/ATS samples (PDF).

\section{AUTHOR INFORMATION}

\section{Corresponding Author}

*E-mail: virginia.martinez@ehu.eus.

\section{ORCID 1}

Yasuhiko Fujita: 0000-0003-1302-1436

Luis Gómez-Hortiguiela: 0000-0002-4960-8709

Virginia Martínez-Martínez: 0000-0001-7551-3714

\section{Notes}

The authors declare no competing financial interest.

\section{ACKNOWLEDGMENTS}

Financial support from Gobierno Vasco (IT912-16) and Ministerio de Economía y Competitividad "MINECO” (through Projects MAT2014-51937-C3-3-P, MAT2016-77496-R and MAT-2015-65767-P) is acknowledged. R.S.L. and V.M.M. acknowledge Universidad del País Vasco (UPV-EHU) for a postdoctoral fellowship and MINECO for a "Ramón y Cajal" Contract (RYC-2011-09505), respectively. H.U. gratefully acknowledges the financial support of the European Research Council (\#280064), the FWO (G056314N, G0B5514N, G081916N), and JSPS KAKENHI (JP17H03003, JP17H05244, JP17H05458). Centro Técnico de Informática (CSIC) is acknowledged for running the calculations and Accelrys for providing the computational software.

\section{REFERENCES}

(1) Hassan Omar, O.; La Gatta, S.; Tangorra, R. R.; Milano, F.; Ragni, R.; Operamolla, A.; Argazzig, R.; Chiorboli, C.; Agostiano, A.; Trotta, M.; Farinola, G. M. Synthetic Antenna Functioning As Light Harvester in the Whole Visible Region for Enhanced Hybrid Photosynthetic Reaction Centers. Bioconjugate Chem. 2016, 27, 1614-1623.

(2) Gartzia-Rivero, L.; Bañuelos, J.; López-Arbeloa, I. Excitation Energy Transfer in Artificial Antennas: From Photoactive Materials to Molecular Assemblies. Int. Rev. Phys. Chem. 2015, 34, 515-556.

(3) Lakowicz, J. R. Principles of Fluorescence Spectroscopy, 3rd ed.; Springer: New York, 2006.

(4) Devaux, A.; Calzaferri, G.; Belser, P.; Cao, P.; Brühwiler, D.; Kunzmann, A. Efficient and Robust Host - Guest Antenna Composite for Light Harvesting. Chem. Mater. 2014, 26, 6878-6885.

(5) Calzaferri, G. Nanochannels: Hosts for the Supramolecular Organization of Molecules and Complexes. Langmuir 2012, 28, 6216-6231.

(6) Calzaferri, G.; Huber, S.; Maas, H.; Minkowski, C. Host - Guest Antenna Materials Angewandte. Angew. Chem., Int. Ed. 2003, 42, 37323758.

(7) Cao, P.; Khorev, O.; Devaux, A.; Sägesser, L.; Kunzmann, A. Supramolecular Organization of Dye Molecules in Zeolite L Channels: Synthesis, Properties, and Composite Materials. Chem. - Eur. J. 2016, 22, 4046-4060.

(8) Cucinotta, F.; Guenet, A.; Bizzarri, C.; Mróz, W.; Botta, C.; MiliánMedina, B.; Gierschner, J.; De Cola, L. Energy Transfer at the Zeolite L Boundaries: Towards Photo- and Electroresponsive Materials. ChemPlusChem 2014, 79, 45-57.

(9) Tabacchi, G.; Fois, E.; Calzaferri, G. Structure of Nanochannel Entrances in Stopcock-Functionalized Zeolite L Composites. Angew. Chem., Int. Ed. 2015, 54, 11112-11116.

(10) Martínez-Martínez, V.; García, R.; Gómez-Hortigüela, L.; Sola Llano, R.; Pérez-Pariente, J.; López-Arbeloa, I. Highly Luminescent and Optically Switchable Hybrid Material by One-Pot Encapsulation of Dyes into MgAPO-11 Unidirectional Nanopores. ACS Photonics 2014, $1,205-211$.
(11) García, R.; Martínez-Martínez, V.; Sola Llano, R.; López-Arbeloa, I. I.; Pérez-Pariente, J. One-Dimensional Antenna Systems by Crystallization Inclusion of Dyes (One-Pot Synthesis) within Zeolitic MgAPO-36 Nanochannels. J. Phys. Chem. C 2013, 117, 24063-24070.

(12) Martínez-Martínez, V.; García, R.; Gómez-Hortigüela, L.; PérezPariente, J.; López-Arbeloa, I. Modulating Dye Aggregation by Incorporation into 1D-MgAPO Nanochannels. Chem. - Eur. J. 2013, 19, 9859-9865.

(13) Prokhorenko, V. I.; Steensgaard, D. B.; Holzwarth, A. R. Exciton Dynamics in the Chlorosomal Antennae of the Green Bacteria Chloroflexus Aurantiacus and Chlorobium Tepidum. Biophys. J. 2000, $79,2105-2120$.

(14) Sato, N.; Fujimura, T.; Shimada, T.; Tani, T.; Tagaki, S. JAggregate Formation Behavior of a Cationic Cyanine Dye on Organic Layered Material. Tetrahedron Lett. 2015, 56, 2902-2905.

(15) Steiger, R.; Pugin, R.; Heier, J. J-Aggregation of Cyanine Dyes by Self-Assembly. Colloids Surf., B 2009, 74, 484-491.

(16) Yao, H. Mesoscopic Morphology, Optical Anisotropy and Spectroscopic Properties of Cyanine J-Aggregates. In J-Aggregates; Kobayashi, T., Ed.; World Scientific: Singapore, 2012; pp 403-441.

(17) Scherer, P. O. J.; Fischer, S. F. On the Theory of Vibronic Structure of Linear Aggregates. Application to Pseudoisocyanin (PIC). Chem. Phys. 1984, 86, 269-283.

(18) Daltrozzo, E.; Scheibe, G.; Gschwind, K.; Haimerl, F. Structure of the J-Aggregates of Pseudoisocyanine. Photogr. Sci. Eng. 1974, 18, 441450 .

(19) von Berlepsch, H.; Böttcher, C.; Dähne, L. Structure of JAggregates of Pseudoisocyanine Dye in Aqueous Solution. J. Phys. Chem. B 2000, 104, 8792-8799.

(20) Forcite Module, Material Studio. Accelrys Inc.: San Diego, CA, 2013.

(21) Baerlocher, C.; McCusker, L. B.; Olson, D. H. Atlas of Zeolite Framework Types; Elsevier, 2007.

(22) García, R.; Martínez-Martínez, V.; Gómez-Hortigüela, L.; López Arbeloa, Í.; Pérez-Pariente, J. Anisotropic Fluorescence Materials: Effect of the Synthesis Conditions over the Incorporation, Alignment and Aggregation of Pyronine Y within MgAPO-5. Microporous Mesoporous Mater. 2013, 172, 190-199.

(23) Choi, S.-E.; Kim, H.; Park, Y.-S.; Lee, J. S. In-Situ Thickness Controlled Growth of AlPO4-5 Films. Microporous Mesoporous Mater. 2016, 219, 155-160.

(24) Sola-Llano, R.; Martínez-Martínez, V.; Fujita, Y.; GómezHortigüela, L.; Alfayate, A.; Uji-i, H.; Fron, E.; Pérez-Pariente, J.; López-Arbeloa, I. Formation of a Nonlinear Optical Host-Guest Hybrid Material by Tight Confinement of LDS 722 into Aluminophosphate 1D Nanochannels. Chem. - Eur. J. 2016, 22, 15700-15711.

(25) McRae, E. G.; Kasha, M.. The Molecular Exciton Model. In Physical Process in Radiation Biology; Augenstein, L., Mason, R., Rosenberg, B., Eds.; Academic Press: New York, 1964; pp 23-42.

(26) Kasha, M.; Rawls, H. R.; El-Bayoumi, M. A. The Exciton Model in Molecular Spectroscopy. Pure Appl. Chem. 1965, 11, 371-392. 